\title{
ITÔ'S FORMULA WITH RESPECT TO FRACTIONAL BROWNIAN MOTION AND ITS APPLICATION
}

\author{
W. DAI \\ Australian National University \\ School of Mathematical Sciences \\ Canberra, ACT 0200, Australia \\ C.C. HEYDE \\ Columbia University \\ 2990 Broadway, Mail Code 4403 \\ New York, NY 10027 USA \\ and \\ Australian National University \\ School of Mathematical Sciences \\ Canberra, ACT 0200, Australia
}

(Received July, 1996; Revised October, 1996)

\begin{abstract}
Fractional Brownian motion (FBM) with Hurst index $1 / 2<H<1$ is not a semimartingale. Consequently, the standard Itô calculus is not available for stochastic integrals with respect to FBM as an integrator if $1 / 2<H<1$. In this paper we derive a version of Itô's formula for fractional Brownian motion. Then, as an application, we propose and study a fractional Brownian Scholes stochastic model which includes the standard Black-Scholes model as a special case and is able to account for long range dependence in modeling the price of a risky asset.
\end{abstract}

This article is dedicated to the memory of Roland L. Dobrushin.

Key words: Fractional Brownian Motion, Itô's Formula, Long Range Dependence, Stochastic Differential Equations, Black-Scholes Model.

AMS (MOS) subject classifications: $60 \mathrm{H} 05,60 \mathrm{H} 10$.

\section{Introduction}

The Itô stochastic calculus has become a fundamental part of modern probability theory and found substantial application in other disciplines. For example, in mathematical finance, Itô's calculus is a powerful tool for dealing with stock price behavior. Stochastic differential equations driven by semimartingales, particularly, Brownian motion, are routinely used to model the dynamics of stock market prices.

A prominent feature of Brownian motion is its independent increments. More generally, however, if $\left\{X_{t}\right\}$ is a stationary process with finite variance and $\gamma_{k}=\operatorname{cov}\left(X_{t}, X_{t+k}\right)$ is the covariance at lag $k$, then $\left\{X_{t}\right\}$ is called short range dependent (SRD) or long range dependent (LRD) according as $\sum_{k=1}^{\infty} \gamma_{k}$ converges or diverges. Equivalently, writing

$$
f(\omega)=\frac{1}{2 \pi} \gamma_{0}+\frac{1}{\pi} \sum_{k=1}^{\infty} \gamma_{k} \cos (k \omega)
$$


for the spectral density of $\left\{X_{t}\right\}$, LRD corresponds to the case where the spectral density tends to infinity as $\omega$ tends to zero and SRD to the case where the spectral density is finite at $\omega=0$.

It is now widely accepted that the assumption of SRD is in various cases, only an approximation to the real LRD structure which occurs in geophysics, hydrology and economics. See, for example, the introduction to the survey article by Beran [1], which includes a general discussion of LRD. The presence of LRD, or the Joseph effect (see, for example, Mandelbrot [10]) is well documented in many economic time series. See, for example, Peters [12] for strong advocacy of the effect of LRD in finance.

Existing models for the dynamics of fluctuating behavior of financial markets are based on the implicit or explicit assumption of SRD, which may not be satisfied in many cases. In a sense, the widely used Black-Scholes model is an extreme case. Some authors (see, for example, Greene and Fielitz [4]; Kunitomo [8]; Peters [12]) have argued that the Black-Scholes model would not be an adequate process for stock price but should be replaced by a model in which the driving process may be LRD.

In order to define a "revised" Black-Scholes model which includes the ordinary Black-Scholes model and is able to account for LRD in stock market price movement, we need to generalize the driving process from SRD to LRD.

Fractional Brownian motion (FBM) provides a suitable generalization of Brownian motion. It is a one-parameter family of Gaussian processes, $B_{H}(t), t \geq 0$ which has zero mean and covariance

$$
E\left[B_{H}(s) B_{H}(t)\right]=\frac{1}{2}\left(|s|^{2 H}+|t|^{2 H}-|t-s|^{2 H}\right) .
$$

Here $0<H<1$ and the case $H=1 / 2$ corresponds to ordinary Brownian motion. FBM arises naturally in a central limit context and from the 1950s it has been proposed as a model for LRD in a variety of hydrological, geophysical and economic time series. See, for example, Hurst [6, 7], Mandelbrot and Van Ness [11], Kunitomo [8] and Gripenberg and Norros [5].

The feature, which most distinguishes FBM from Brownian motion, is that FBM is no longer a semimartingale for $1 / 2<H<1$ (e.g., Lin [9]). This necessitates a careful definition of the stochastic integral with respect to FBM from first principles. See, for example, Gripenberg and Norros [5], Lin [9] and Dai and Heyde [3] for contributions to this subject. For the purpose of defining stochastic differential equations driven by FBM, it is necessary to derive the corresponding Itô's formula with respect to FBM. We turn to this matter in Section 3 . In Section 4, a plausible counterpart to the now-classical Black-Scholes model is suggested. Then we use the results of Section 3 to prove the existence and uniqueness of the solution of the fractional BlackScholes stochastic differential equation.

\section{Definition of Stochastic Differential Equations Driven by Fractional Brownian Motion}

In this section, we are concerned with the definition of stochastic differential equations with respect to FBM. Several different ways of a defining stochastic integral with respect to FBM have been suggested. See, for example, Gripenberg and Norros [5], Lin [9] and Dai and Heyde [3]. we use the definition given by Dai and Heyde. Here we assume that $(\Omega, \mathscr{F}, P)$ is complete probability space associated with a standard normalized FBM $B_{H}(t)$ on a finite interval $[0, T]$. We further assume that $1 / 2<H<1$.

Definition 1: Let $a(t, \omega)$ and $b(t, \omega):[0, T] \times \Omega \rightarrow \mathbf{R}$ be two stochastic processes. We say that a stochastic process $\{X(t): t \in[0, T]\}$ has a stochastic differential with respect to fractional Brown- 
ian motion $B_{H}(t)$

$$
d X_{t}=a(t) d t+b(t) d B_{H}(t)
$$

if for any $(t, \omega) \in[0, T] \times \Omega$, the following holds

$$
X(t, \omega)=X_{0}(\omega)+\int_{0}^{t} a(s, \omega) d s+\int_{0}^{t} b(s, \omega) d B_{H}(s, \omega),
$$

where $X_{0}$ is a random variable. The stochastic integral $\int_{0}^{t} a(s, \omega) d s$ is an ordinary RiemannStieltjes integral for each $\omega \in \Omega$ while $\int_{0}^{t} b(s, \omega) d B_{H}(s, \omega)$ is defined as that given by Dai and
Heyde [3].

Remarks on Definition 1: Generally speaking, the integral $\int_{0}^{t} a(s, \omega) d s$ exists under standard conditions on $a(s, \omega)$. The integral $\int_{0}^{t} b(s, \omega) d B_{H}(s, \omega)$ exists only under the conditions given in Dai and Heyde [3] for defining stochastic integrals with respect to $B_{H}(t)$. We will discuss equation (1) in more detail in Section 3.

Definition 2: (Fractional Black-Scholes model). The stochastic differential equation

$$
d S_{t}=\mu S_{t} d t+\sigma S_{t} d B_{H}(t)
$$

is called a fractional Black-Scholes model, where $\mu$ and $\sigma$ are constants and the Hurst index satisfies $1 / 2 \leq H<1$.

Remarks on Definition 2: When $H=1 / 2,(3)$ is the well known Black-Scholes model. Since the Black-Scholes model has been studied thoroughly, we concentrate here on the case where $1 / 2<H<1$. We discuss equation (3) in Section 4 .

\section{Itô's Formula with Respect to Fractional Brownian Motion}

When we consider stochastic differential equations driven by Brownian motion

$$
d X_{t}=a\left(t, X_{t}\right) d t+B\left(t, X_{t}\right) d B(t),
$$

Itô's formula is a powerful tool for dealing with their calculus. When we are concerned with stochastic differential equations driven by fractional Brownian motion

$$
d X_{t}=a\left(t, X_{t}\right) d t+b\left(t, X_{t}\right) d B_{H}(t)
$$

we have noticed that a version of Itô's formula plays the same role in dealing with equation (5). The aim of this section is the following theorem.

Theorem 1: (Itô's formula with respect to fractional Brownian motion) Let $(\Omega, \mathcal{F}, P)$ be a complete probability space. Let $B_{H}(\tau)$ be a fractional Brownian motion on $[0, T]$ such that $1 / 2<H<1$ and $B_{H}(0)=0$ a.e. (therefore $E B_{H}(\tau) \equiv 0$ for any $\left.\tau \in[0, T]\right)$. Assume stochastic processes $a(\tau, \omega), b(\tau, \omega)$ and $X(\tau, \omega)$ are such that for any $\left[t_{0}, t\right] \subseteq[0, T]$,

1. $\quad a(\tau, \omega)$ is Riemann-Stieltjes integrable on $\left[t_{0}, t\right]$ for each $\omega \in \Omega$;

2. $\quad \int_{0}^{t} b(\tau) d B_{H}(\tau)$ exists in the sense described in Dai and Heyde [3];

3. Either of the following holds

$$
\begin{aligned}
& \text { for any } 0 \leq s \leq t_{1} \leq t_{2}, t_{3} \leq t_{4} \leq T, \quad\{b(\tau): 0 \leq \tau \leq T\} \quad \text { and } \quad\left\{B_{H}(\tau): 0 \leq \tau \leq T\right\} \\
& \text { are such that }
\end{aligned}
$$




$$
\begin{gathered}
\\
\left\{E \left(\left(b\left(t_{1}\right)-b(s)\right)\left(b\left(t_{3}\right)-b(s)\right)\left(B_{H}\left(t_{2}\right)-B_{H}\left(t_{2}\right)\right)\left(B_{H}\left(t_{4}-B_{H}\left(t_{4}\right)\right)\right\}\right.\right. \\
=\left\{E\left(\left(b\left(t_{1}\right)-b(s)\right)\left(b\left(t_{3}\right)-b(s)\right)\right\} E\left\{\left(B_{H}\left(t_{2}\right)-B_{H}\left(t_{2}\right)\right)\left(B_{H}\left(t_{4}\right)-B_{H}\left(t_{4}\right)\right)\right\}\right.
\end{gathered}
$$

or,

3.2 the second derivative $d^{2} b(t) / d t^{2}$ exists, and for any $0 \leq s \leq t_{1} \leq t_{2}, t_{3} \leq t_{4} \leq T$, $\left\{b^{\prime}(\tau)=d b(\tau) / d \tau: s \leq \tau \leq \max \left\{t_{1}, t_{3}\right\}\right\}$ and $\left(B_{H}\left(t_{1}\right), B_{H}\left(t_{2}\right), B_{H}\left(t_{3}\right), B_{H}\left(t_{4}\right)\right)$ are such that for any random variables $\xi$ and $\eta$ such that $\xi$ and $\eta$ are measurable with respect to $\sigma\left\{b^{\prime}(\tau): s \leq \tau \leq \max \left\{t_{1}, t_{3}\right\}\right\}$ and $E|\xi|^{4}<\infty, E|\eta|^{4}<\infty$, the following holds

$$
\begin{gathered}
\left.\left.\left.E\left\{\left(b^{\prime}(s) t_{1}-s\right)+\xi\right)\right)\left(b^{\prime}(s)\left(t_{3}-s\right)+\eta\right)\right)\left(B_{H}\left(t_{2}\right)-B_{H}\left(t_{1}\right)\right)\left(B_{H}\left(t_{4}\right)-B_{H}\left(t_{3}\right)\right)\right\} \\
\left.\left.E\left\{\left(b^{\prime}(s)\left(t_{1}-s\right)+\xi\right)\right)\left(b^{\prime}(s)\left(t_{3}-s\right)+\eta\right)\right)\right\} E\left\{\left(B_{H}\left(t_{2}\right)-B_{H}\left(t_{1}\right)\right)\left(B_{H}\left(t_{4}\right)-B_{H}\left(t_{3}\right)\right)\right\}
\end{gathered}
$$

and furthermore,

$$
\sup _{0 \leq t \leq T} E\left|\frac{d b}{d t}(t, \omega)\right|^{4}<\infty, \sup _{0 \leq t \leq T} E\left|\frac{d^{2} b}{d t^{2}}(t, \omega)\right|^{4}<\infty
$$

4.

$$
X_{t}-X_{t_{0}}=\int_{t_{0}}^{t} a(\tau, \omega) d \tau+\int_{t_{0}}^{t} b(\tau, \omega) d B_{H}(\tau),
$$

where the first integral in (9) is an ordinary Riemann-Stieltjes integral for each $\omega \in \Omega$, while the second is an Itô integral defined in Dai and Heyde [3]. Assume that a two variable function $U(t, x):[0, T] \times \mathbf{R} \rightarrow \mathbf{R}$ has uniformly continuous partial derivatives $\partial U / \partial t, \partial U / \partial x$ and $\partial^{2} U / \partial x^{2}$. Assume further that

$$
\begin{gathered}
\sup _{0 \leq t \leq T} E\left|U\left(t, X_{t}\right)\right|^{2}<\infty \\
\sup _{0 \leq t \leq T} E\left|\frac{\partial U}{\partial t}\left(t, X_{t}\right)\right|^{2}<\infty \\
\sup _{0 \leq t \leq T} E\left|\frac{\partial U}{\partial x}\left(t, X_{t}\right)\right|^{2}<\infty \\
\sup _{0 \leq t \leq T} E\left|\frac{\partial^{2} U}{\partial x^{2}}\left(t, X_{t}+O_{L_{2}}(1)\right)\right|^{2}<\infty \\
\sup _{0 \leq t \leq T} E|a(t)|^{2}<\infty \\
\sup _{0 \leq t \leq T} E|b(t)|^{2}<\infty \\
E|b(t)-b(s)|<\text { const }|t-s|^{\beta}, \beta \geq 0
\end{gathered}
$$

where $O_{L_{2}}(1)$ means a term such that $E\left|O_{L_{2}}(1)\right|^{2}<\infty$. Let $U_{t}=U\left(t, X_{t}\right)$. If, for any $0 \leq t \leq T$,

$$
\int_{0}^{t} b(\tau, \omega) \frac{\partial U}{\partial x}\left(\tau, X_{\tau}\right) d B_{H}(\tau)
$$


exists in the sense described in Dai and Heyde [3], then the following holds

or equivalently,

$$
\begin{aligned}
Y_{t}=Y_{t_{0}} & =\int_{0}^{t}\left\{\frac{\partial U}{\partial t}\left(\tau, X_{\tau}\right)+a(\tau, \omega) \frac{\partial U}{\partial x}\left(\tau, X_{\tau}\right\} d \tau\right. \\
& +\int_{t_{0}}^{t} b(\tau, \omega) \frac{\partial U}{\partial x}\left(\tau, X_{\tau}\right) d B_{H}(\tau),
\end{aligned}
$$

$$
d Y_{t}=\left\{\frac{\partial U}{\partial t}\left(t, X_{t}\right)+a(t, \omega) \frac{\partial U}{\partial x}\left(t, X_{t}\right)\right\} d t+b(t, \omega) \frac{\partial U}{\partial x}\left(t, X_{t}\right) d B_{H}(t) .
$$

\section{Remarks on Theorem 1:}

1. Since $E\left(B_{H}(t+\Delta)-B_{H}(t)\right)^{2}=|\Delta|^{2 H}$, where $2 H>1$, there is no term

$$
\frac{1}{2} b^{2}(\tau, \omega) \frac{\partial^{2} U}{\partial x^{2}}\left(\tau, X_{t}\right) d \tau
$$

in (17), in contrast to that of the usual Itô formula with respect to Brownian motion.

2. The requirements on $(\tau), b(\tau), X(\tau)$ and $U\left(\tau, X_{\tau}\right)$, such as Conditions 1,2 and 4 of the theorem, and the moment conditions (10)-(15) are standard.

3. Conditions 3.1 and 3.2 are important for Itô's formula to be true in the case of fractional Brownian motion. Many stochastic processes can be chosen as $b(\tau)$. For example,

$$
b(\tau)=A_{1} \tau+A_{2},
$$

where $A_{1}$ and $A_{2}$ are two random variable with $E A_{1}^{2}<\infty$ and $A_{1}$ is independent of $\left\{B_{H}(\tau)\right\}$.

The proof of Theorem 1 will be given in Section 5 .

\section{Application of Stochastic Calculus of Fractional Brownian Motion}

\subsection{Summary of some other results on stochastic calculus of $B_{H}(t)$}

A number of authors have been interested in the stochastic analysis of $B_{H}(t)$. For example, Lin [9] defined the stochastic integral with respect to $B_{H}(t)$ in the case where the integrands are either deterministic bounded functions or the compositions of deterministic bounded functions and $B_{H}(t)$. He also investigated stochastic differential equations of the form

$$
d X_{t}=f\left(t, X_{t}\right) d t+g(t) d B_{H}(t) .
$$

In this subsection we summarize some of his results.

Definition 3: Let $g(t): \mathbf{R} \rightarrow \mathbf{R}$ be a bounded Borel function. Define

$$
\begin{gathered}
\int_{0}^{t} g\left(B_{H}(\tau)\right) d B_{H}(\tau)=\int_{0}^{B_{H}(t)} g(\tau) d \tau, \\
\int_{0}^{B_{H}(t)} g(\tau) d \tau=\lim _{\left|\beta^{n}\right| \rightarrow 0} \sum_{j} g\left(t_{j-1}^{n}\right)\left(B_{H}\left(t_{j}^{n}\right)-B_{H}\left(t_{j-1}^{n}\right)\right),
\end{gathered}
$$

where the sequence of partitions of $[0, t]$ is given as the same as that of Dai and Heyde [3]. 
Remarks on Definition 3: Definition 20 is a special case of Definition 7 of Dai and Heyde [3], while (21) is a special case of Definition 6 of Dai and Heyde [3]. Lin studied the existence and uniqueness of equation (19). He found the following result.

Theorem 2: (Lin, [9]) Let $f(s, x)$ and $g(s)$ be Borel functions such that

1. $g:[0, \infty) \rightarrow \mathbf{R}$ is bounded,

2. $\quad|f(s, x)| \leq K|x|+K$,

3. $\quad|f(s, x)-f(s, y)| \leq K|x-y|$.

Here $K$ is a positive constant. Then the stochastic differential equation

$$
\left\{\begin{array}{c}
d X_{t}=f\left(t, X_{t}\right) d t+g(s) d B_{H}(t) \\
X_{0}=A(\omega)
\end{array}\right.
$$

has a unique solution, whose paths are continuous. Here $A(\omega) \in L_{2}(\Omega)$.

For the proof of Theorem 2, see Lin [9].

\subsection{The existence and uniqueness of the solution of the fractional Black-Scholes equation}

In this subsection, we are interested in solving a stochastic differential equation - the fractional Black-Scholes model defined in Section 2. We will use Itô's formula (18) and Theorem 2 to prove the uniqueness of the solution of the fractional Black-Scholes equation (3). In detail, we have the following theorems:

Theorem 3: The stochastic differential equation

has a solution

$$
\left\{\begin{array}{c}
d S_{t}=\mu S_{t} d t+\sigma S_{t} d B_{H}(t) \\
S_{t_{0}}=A(\omega)
\end{array}\right.
$$

$$
S_{t}=A \exp \left\{\mu\left(t-t_{0}\right)+\sigma\left(B_{H}(t)-B_{H}\left(t_{0}\right)\right)\right\}
$$

where $A(\omega)$ is a positive random variable, such that $E|A(\omega)|^{2}<\infty, \mu$ and $\sigma$ are constants.

Theorem 4: The solution of (23) is unique.

Remarks on Theorems 3 and 4: We derived a solution of (23) before we read the work of Lin. Subsequently, we have used his result (Theorem 2) to prove the uniqueness of (23). The original method we used to prove Theorem 3 is given in Subsection 5.5.2, Dai [2]. Here we use the result of Lin to show the existence and uniqueness through Theorems 3 and 4.

Proof of Theorems 3 and 4: Let us consider a stochastic equation

$$
\left\{\begin{array}{c}
d X_{t}=\mu d t+\sigma d B_{H}(t) \\
X_{t_{0}}=\log A
\end{array}\right.
$$

where $\mu, \sigma$ and $A$ are as given in Theorem 3. Then it is easy to see that

$$
X_{t}=X_{t_{0}}+\mu\left(t-t_{0}\right)+\sigma\left(B_{H}(t)-B_{H}\left(t_{0}\right)\right)
$$

is a solution of (25) and furthermore, from Theorem 2 , it is the unique solution. Now let

$$
S_{t}=\exp \left\{X_{t}\right\}
$$

then by Itô's formula (18) we have

$$
\begin{gathered}
d S_{t}=\mu \exp \left\{X_{t}\right\} d t+\sigma \exp \left\{X_{t}\right\} d B_{H}(t) \\
=\mu S_{t} d t+\sigma S_{t} d B_{H}(t),
\end{gathered}
$$


therefore,

$$
S_{t}=A \exp \left\{\mu\left(t-t_{0}\right)+\sigma\left(B_{H}(t)-B_{H}\left(t_{0}\right)\right)\right\}
$$

is the unique solution of equation (23).

\section{Proof of Theorem 1}

In order to prove Theorem 1, we need the following lemma, the proof of which will be given after the proof of Theorem 1.

Lemma 5: Assume stochastic processes $a(\tau)$ and $b(\tau)$ satisfy the conditions of Theorem 1. Then, for any $t, s \in[0, T]$ such that $|t-s| \rightarrow 0$, we have

$$
\int_{s}^{t} a(\tau) d \tau+\int_{s}^{t} b(\tau) d B_{H}(\tau)=a(s)(t-s)+b(s)\left(B_{H}(t)-B_{H}(s)\right)+o_{L_{2}}(|t-s|)
$$

where $o_{L_{2}}(|t-s|)$ means a term such that

$$
\left(E\left|o_{L_{2}}(|t-s|)\right|^{2}\right)^{1 / 2}=o(|t-s|)
$$

Proof of Theorem 1: For any interval $\left[t_{0}, t\right] \subseteq[0, T]$ and any sequence of partitions

$$
\beta^{(n)}: t_{0}=t_{0}^{(n)}<t_{1}^{(n)}<\ldots<t_{q(n)}^{(n)}=t
$$

with $\left|\beta^{(n)}\right| \rightarrow 0$ as $n \rightarrow \infty$, write

$$
\begin{gathered}
\Delta t_{j}^{(n)}=t_{j+1}^{(n)}-t_{j}^{(n)}, \Delta X_{j}^{(n)}=X_{t_{j+1}^{(n)}}-X_{t_{j}^{(n)}}, \\
\Delta B_{H, j}^{(n)}=B_{H}\left(t_{j+1}^{(n)}\right)-B_{H}\left(t_{j}^{(n)}\right), \\
\Delta U_{j}^{(n)}=U\left(t_{j+1}^{(n)}, X_{t_{j+1}^{(n)}}\right)-U\left(t_{j}^{(n)}, X_{t_{j}^{(n)}}\right)
\end{gathered}
$$

for $j=0,1, \ldots, q(n)-1, n=1,2, \ldots$. Then we have

$$
Y_{t}-Y_{t_{0}}=U\left(t, X_{t}\right)-U\left(t_{0}, X_{t_{0}}\right)=\lim _{n \rightarrow \infty} \sum_{j=0}^{q(n)} \Delta U_{j}^{(n)} .
$$

From a knowledge of calculus we have

$$
\begin{aligned}
\Delta U_{j}^{(n)}=\frac{\partial U}{\partial t} & \left.t_{j}^{(n)}+\theta_{n} \Delta t_{j}^{(n)}, X_{t_{j+1}^{(n)}}\right) \Delta t_{j}^{(n)}+\frac{\partial U}{\partial x}\left(t_{j}^{(n)}, X_{t_{j}^{(n)}}\right) \Delta X_{j}^{(n)} \\
& +\frac{\partial^{2} U}{\partial x^{2}}\left(t_{j}^{(n)}, X_{t_{j}^{(n)}}+\delta_{n} \Delta X_{j}^{(n)}\right)\left(\Delta X_{j}^{(n)}\right)^{2}
\end{aligned}
$$

where $\theta_{n}=\theta_{n}(\omega)$ and $\delta_{n}=\delta_{n}(\omega)$ are random variable such that $0 \leq \theta_{n}, \delta_{n} \leq 1$ and $\lim _{n \rightarrow \infty} \theta_{n}=\lim _{n \rightarrow \infty} \delta_{n}=0$ in the $L_{2}(\Omega)$ sense. Since $\partial U / \partial x$ is uniformly continuous and the stochastic process $X_{t}$ is continuous in the sense of $L_{2}(\Omega)$ (as well as with probability one, see Theorem 16, Dai and Heyde [3]), we have

$$
\lim _{n \rightarrow \infty} \sum_{j=0}^{q(n)} \frac{\partial U}{\partial t}\left(t_{j}^{(n)}+\theta_{n} \Delta t_{j}^{(n)}, X_{t_{j+1}^{(n)}}\right) \Delta t_{j}^{(n)}=\int_{t_{0}}^{t} \frac{\partial U}{\partial t}\left(\tau, X_{\tau}\right) d \tau .
$$

From Lemma 5 and (9) we have

$$
\Delta X_{j}^{(n)}=a\left(t_{j}^{(n)}\right) \Delta t_{j}^{(n)}+b\left(t_{j}^{(n)}\right) \Delta B_{H, j}^{(n)}+o_{L_{2}}\left(\Delta t_{j}^{(n)}\right),
$$


where $o_{L_{2}}\left(\Delta t_{j}^{(n)}\right)$ means a term such that

$$
\left(E\left|o_{L_{2}}\left(\Delta t_{j}^{(n)}\right)\right|^{2}\right)^{1 / 2}=o\left(\Delta t_{j}^{(n)}\right)
$$

Therefore, by (12),

$$
\begin{gathered}
\sum_{j=0}^{q(n)-1} \frac{\partial U}{\partial x}\left(t_{j}^{(n)}, X_{t_{j}^{(n)}}\right) \Delta X_{j}^{(n)} \\
=\sum_{j=0}^{q(n)-1} \frac{\partial U}{\partial x}\left(t_{j}^{(n)}, X_{t_{j}^{(n)}}\right)\left\{a\left(t_{j}^{(n)}\right) \Delta t_{j}^{(n)}+b\left(t_{j}^{(n)}\right) \Delta B_{H, j}^{(n)}\right\}+o_{L_{2}}(1),
\end{gathered}
$$

where $o_{L_{2}}(1)$ means a term such that $\lim _{n \rightarrow \infty} E\left|o_{L_{2}}(1)\right|^{2}=0$. Hence

$$
\begin{aligned}
& \lim _{n \rightarrow \infty} \sum_{j=0}^{q(n)-1} \frac{\partial U}{\partial x}\left(t_{j}^{(n)}, X_{t_{j}^{(n)}}\right) \Delta X_{j}^{(n)} \\
= & \int_{t_{0}}^{t} \frac{\partial U}{\partial x}\left(\tau, X_{\tau}\right)\left\{a(\tau)+b(\tau) d B_{H}(\tau)\right\} .
\end{aligned}
$$

From Lemma 5, (14), (15) and noticing that

we have

$$
E\left(B_{H}(t+\tau)-B_{H}(t)\right)^{2}=\tau^{2 H} V_{H}
$$

Then, by (13),

$$
\left(\Delta X_{j}^{(n)}\right)^{2}=o_{L_{2}}\left(\Delta t_{j}^{(n)}\right) .
$$

$$
\frac{\partial^{2} U}{\partial x^{2}}\left(t_{j}^{(n)}, X_{t_{j}^{(n)}}+\delta_{n} \Delta X_{j}^{(n)}\right)\left(\Delta X_{j}^{(n)}\right)^{2}=o_{L_{2}}\left(\Delta t_{j}^{(n)}\right)
$$

and hence

$$
\lim _{n \rightarrow \infty} \sum_{j=0}^{q(n)-1} \frac{\partial^{2} U}{\partial x^{2}}\left(t_{j}^{(n)}, X_{t_{j}^{(n)}}+\delta_{n} \Delta X_{j}^{(n)}\right)\left(\Delta X_{j}^{(n)}\right)^{2}=0
$$

Now, from (27), (28), (29), (30) and (31), we have

$$
Y_{t}-Y_{t_{0}}=\int_{0}^{t}\left\{\frac{\partial U}{\partial t}\left(\tau, X_{\tau}\right)+\frac{\partial U}{\partial x}\left(\tau, X_{\tau}\right) a(\tau)\right\} d \tau+\int_{0}^{t} \frac{\partial U}{\partial x}\left(\tau, X_{\tau}\right) b(\tau) d B_{H}(\tau)
$$

This finishes the proof of Theorem 1. Next we move to establish Lemma 5.

Proof of Lemma 5: Since $a(t, \omega)$ is Riemann-Stieltjes integrable, as $|t-s| \rightarrow 0$, from Lemma 16 of Dai and Heyde [3], we have

$$
\int_{s}^{t} a(\tau) d \tau=a(s)(t-s)+o_{L_{2}}(|t-s|)
$$

Hence, in order to finish the proof of Lemma 5 , we need only to show that

$$
\int_{s}^{t} b(\tau) d B_{H}(\tau)=b(s)\left(B_{H}(t)-B_{H}(s)\right)+o_{L_{2}}(|t-s|) .
$$

Without loss of generality, we assume $s<t$. Let a sequence of partitions of $[s, t]$ be given as 
then

$$
\beta^{(n)}: s=t_{0}^{(n)}<t_{1}^{(n)}<\ldots<t_{q(n)}^{(n)}=t
$$

$$
\begin{gathered}
E\left|\int_{s}^{t} b(\tau) d B_{H}(\tau)-b(s)\left(B_{H}(t)-B_{H}(s)\right)\right|^{2} \\
=\lim _{n \rightarrow \infty} E\left|\sum_{j=1}^{n}\left(b\left(t_{j-1}^{(n)}\right)-b(s)\right)\left(B_{H}\left(t_{j}^{(n)}\right)-B_{H}\left(t_{j-1}^{(n)}\right)\right)\right|^{2} .
\end{gathered}
$$

Now we consider the term on the right-hand side of (33) without taking the limit yet. We have

$$
\begin{gathered}
E\left|\sum_{j=1}^{n}\left(b\left(t_{j-1}^{(n)}\right)-b(s)\right)\left(B_{H}\left(t_{j}^{(n)}\right)-B_{H}\left(t_{j-1}^{(n)}\right)\right)\right|^{2} \\
=\sum_{j, k=1}^{n} E\left(b\left(t_{j-1}^{(n)}\right)-b(s)\right)\left(b\left(t_{k-1}^{(n)}\right)-b(s)\right)\left(B_{H}\left(t_{j}^{(n)}\right)-B_{H}\left(t_{j-1}^{(n)}\right)\right)\left(B_{H}\left(t_{k}^{(n)}\right)-B_{H}\left(t_{k-1}^{(n)}\right)\right) \\
\equiv A_{n}+B_{n}, \text { say. }
\end{gathered}
$$

If Condition 3.1 of Theorem 1 holds, then by (16),

$$
\begin{gathered}
A_{n}=\sum_{j=1}^{n} E\left(b\left(t_{j-1}^{(n)}\right)-b(s)\right)^{2} E\left(B_{H}\left(t_{j}^{(n)}\right)-B_{H}\left(t_{j-1}^{(n)}\right)\right)^{2} \\
\leq \text { const }|t-s|^{\beta+2 H}=o(|t-s|) .
\end{gathered}
$$

To deal with $B_{n}$ in (34) under Condition 3.1 of Theorem 1, we use the notation $\Gamma_{j, k}, \Delta \Gamma_{j, k}$ and $\alpha$ appearing in Lemma 21 of Dai and Heyde [3]. Since $\left|\partial^{2} \Gamma / \partial y \partial x\right|$ is integrable in $\{(x, y)$ : $s \leq x \neq y \leq t\}$, by Lemma 21, Dai and Heyde [3], (16) and the Cauchy-Schwarz inequality, we have

$$
\begin{gathered}
B_{n}=\sum_{j \neq k}\left\{E\left(b\left(t_{j-1}^{(n)}\right)-b(s)\right) b\left(t_{k-1}^{(n)}\right)-b(s)\right\} \Delta \Gamma_{j, k} \\
\leq \text { const }|t-s|^{\beta} \sum_{j \neq k}\left\{\left|\frac{\partial^{2} \Gamma}{\partial y \partial x}\left(t_{j-1}^{(n)}, t_{k-1}^{(n)}\right)\right|\left(t_{j}^{(n)}-t_{j-1}^{(n)}\right)\left(t_{k}^{(n)}-t_{k-1}^{(n)}\right)\right. \\
\left.+C\left|t_{j-1}^{(n)}-t_{k-1}^{(n)}\right|^{2 H-2-\alpha} o\left(\left(t_{j}^{(n)}-t_{j-1}^{(n)}\right)\left(t_{k}^{(n)}-t_{k-1}^{(n)}\right)\right)+o\left(\left(t_{j}^{(n)}-t_{j-1}^{(n)}\right)\left(t_{k}^{(n)}-t_{k-1}^{(n)}\right)\right)\right\} \\
\rightarrow \text { const }|t-s|^{\beta} \iint_{[s, t]^{2}}\left|\frac{\partial^{2} \Gamma}{\partial y \partial x}(x y)\right| d y d x=o(|t-s|) .
\end{gathered}
$$

So, in the case of Condition 3.1, from (34), (35) and (36), Lemma 5 holds. Finally, we consider the case of Condition 3.2. Following (34) and using the inequalities of Condition 3.2, we have

$$
\begin{gathered}
A_{n}=\sum_{j=1}^{n} E\left\{\left(b^{\prime}(s)\left(t_{j-1}^{(n)}-s\right)+o_{L_{4}}\left(t_{j-1}^{(n)}-s\right)\right)^{2}\left(B_{H}\left(t_{j}^{(n)}\right)-B_{H}\left(t_{j-1}^{(n)}\right)\right)^{2}\right\} \\
\leq \operatorname{const}(t-s)^{2} \sum_{j=1}^{q(n)}\left(t_{j}^{(n)}-t_{j-1}^{(n)}\right)^{2 H}=o(t-s) .
\end{gathered}
$$

By the same argument, under Condition 3.2 , we have

$$
B_{n}=\leq \text { const }|t-s|^{2} \sum_{j \neq k}\left|\Delta \Gamma_{j, k}\right|=\text { const }|t-s|^{2+2 H}=o(t-s) .
$$


Thus, in the case of Condition 3.2, from (34), (37) and (38), Lemma 5 holds. This completes the proof of Lemma 5 , and hence Theorem 1.

\section{References}

[1] Beran, J., Statistical models for data with long-range dependence, Statistical Science 7 (1992), 404-427.

[2] Dai, W., Long range dependent processes and fractional Brownian motion, Ph.D. thesis, Australian National University (1996).

[3] Dai, W. and Heyde, C.C., Stochastic integrals with respect to fractional Brownian motion, (1996), preprint.

[4] Greene, M.T. and Fielitz, B.D., Long-term dependence in common stock returns, J. Financial Econom. 4 (1977), 339-349.

[5] Gripenberg, G. and Norros, I., On the prediction of fractional Brownian motion, J. Appl. Prob. 33 (1996), 400-410.

[6] Hurst, H.E., Long-term storage capacity of reservoirs, Trans. Amer. Soc. Civil Eng. 116 (1951), 400-410.

[7] Hurst, H.E., Methods of using long-term storage in reservoirs, Proc. Inst. Civil Engineers Part I, Chapter 5 (1956), 519-590.

[8] Kunitomo, N., Long-term memory and fractional Brownian motion in financial markets, (1993), preprint.

[9] Lin, S.J., Stochastic analysis of fractional Brownian motions, Stochastics, to appear.

[10] Mandelbrot, B.B., When can price be arbitraged efficiently? A limit to the validity of the random walk and martingale models, Rev. of Economics and Statistics 53 (1971), 225-236.

[11] Mandelbrot, B.B. and Van Ness, J.W., Fractional Brownian motions, fractional noises and applications, SIAM Rev. 10 (1968), 422-437.

[12] Peters, E.E., Fractal Market Analysis, Wiley, New York 1994. 


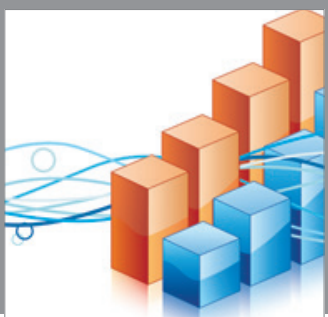

Advances in

Operations Research

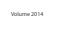

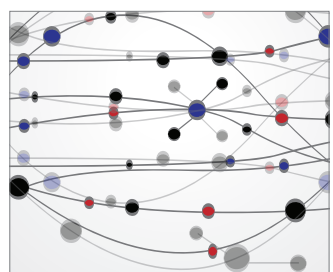

\section{The Scientific} World Journal
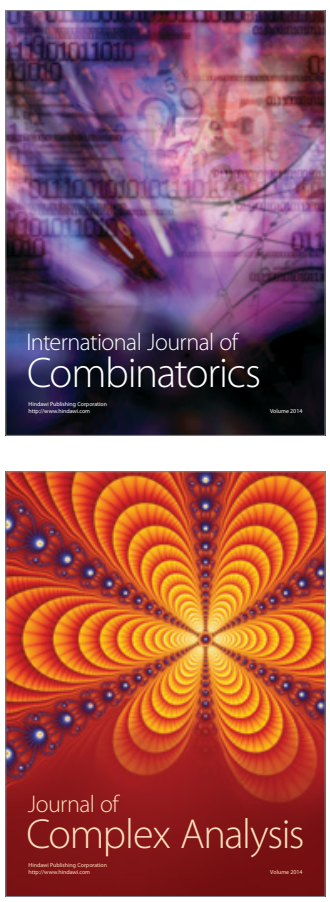

International Journal of

Mathematics and

Mathematical

Sciences
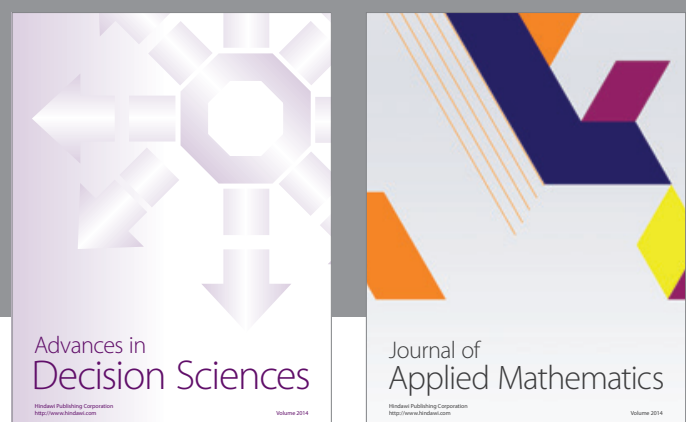

Journal of

Applied Mathematics
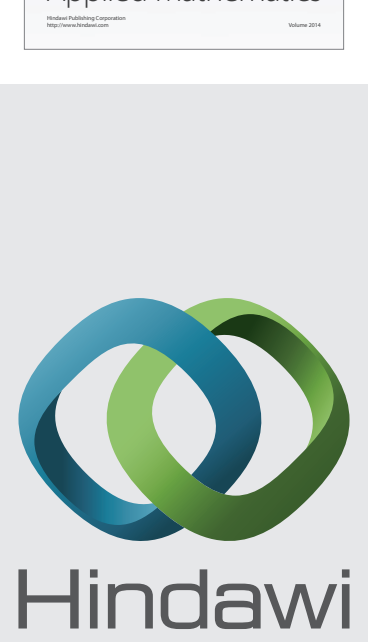

Submit your manuscripts at http://www.hindawi.com
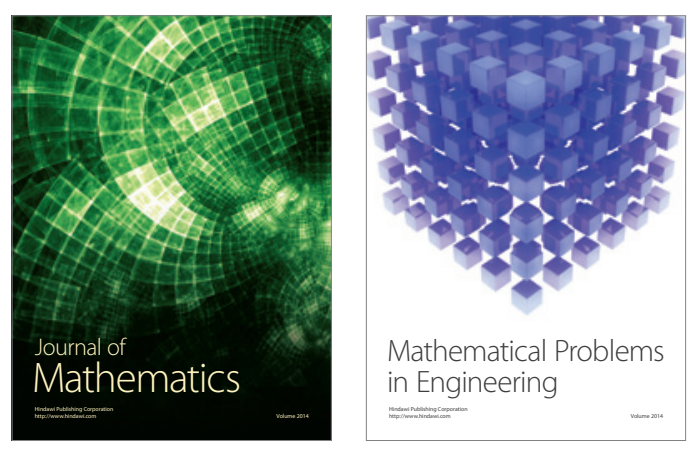

Mathematical Problems in Engineering
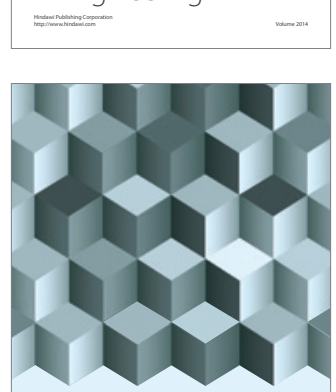

Journal of

Function Spaces
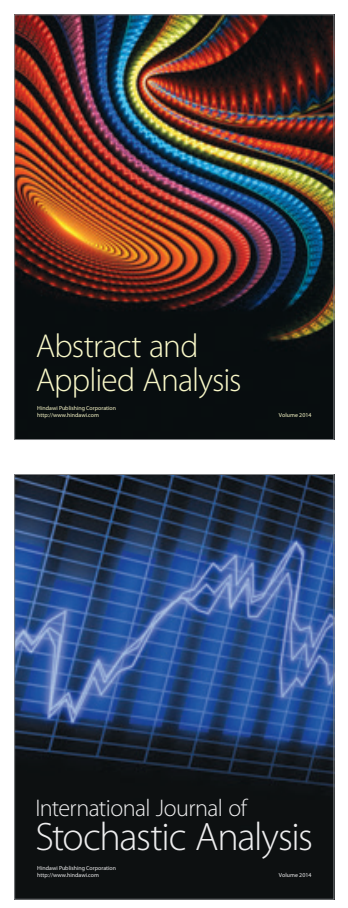

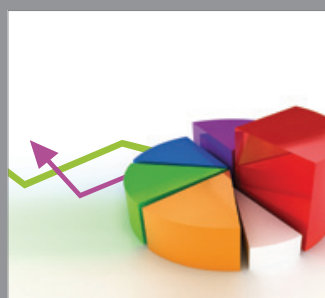

ournal of

Probability and Statistics

Promensencen
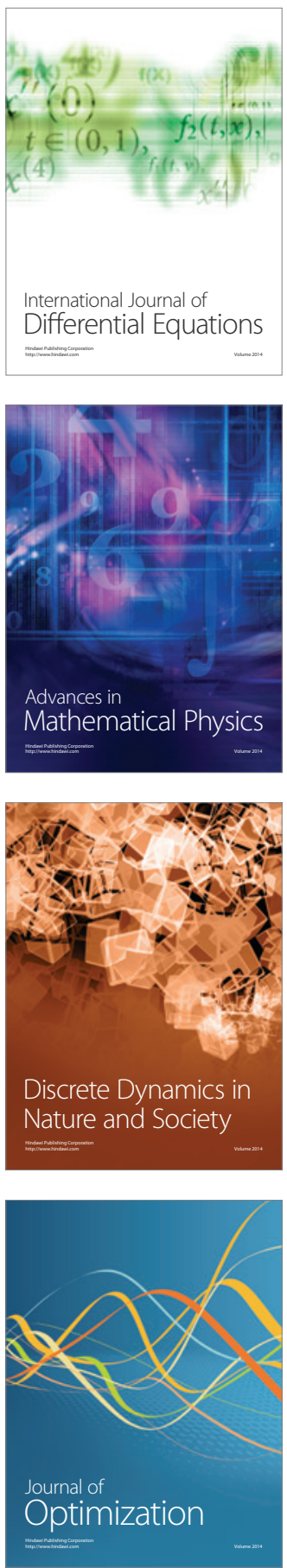